\title{
ON THE RINGS OF FORMAL SOLUTIONS OF POLYNOMIAL DIFFERENTIAL EQUATIONS
}

\author{
MARIA-ANGELES ZURRO \\ Departamento de Matemáticas, Facultad de Ciencias \\ Universidad Autónoma de Madrid \\ Ciudad Universitaria de Canto Blanco, 28049 Madrid, Spain \\ E-mail: mangeles.zurro@uam.es
}

Dedicated to Professor S. Eojasiewicz on his seventieth birthday

\begin{abstract}
The paper establishes the basic algebraic theory for the Gevrey rings. We prove the Hensel lemma, the Artin approximation theorem and the Weierstrass-Hironaka division theorem for them. We introduce a family of norms and we look at them as a family of analytic functions defined on some semialgebraic sets. This allows us to study the analytic and algebraic properties of this rings.
\end{abstract}

Introduction. The Gevrey series appeared as formal solutions of partial differential equations of second order $([\mathrm{G}])$. In 1903 Maillet ([Mi]) proved that the formal solutions of ordinary differential equations with polynomial coefficients are of this type. Afterwards, Malgrange $([\mathrm{Ml}])$ and J. Cano $([\mathrm{Ca}])$ generalized this result to ordinary differential equations with analytic and Gevrey coefficients respectively. But for partial differential equations the analogous result is not yet achieved for any partial differential equation with polynomial coefficients; nevertheless there are some important results: see Ouchi $([\mathrm{O}])$.

The semianalytic geometry with Gevrey conditions to the border is studied in the article of Tougeron $[\mathrm{T}]$. He generalized the basic theorems of the semianalytic geometry to this case.

As far as we know, a study of the algebraic properties of this series has not yet been done. This work shows the basic algebraic properties of these rings. In particular, they are noetherian and henselian. The basic tools we use here are the pseudo-Banach structure they have, and the formal Borel transform. This transform changes the Gevrey series by

1991 Mathematics Subject Classification: 13J, 13H, 32A05, 35A05.

Received by the editors: January 20, 1997; in the revised form July 29, 1997.

The paper is in final form and no version of it will be published elsewhere. 
a germ of analytic function at 0 . We introduce a family of norms that are the restrictions of the absolute Borel transform to the semialgebraic $(0, \infty)^{n} \cap\left\{x_{1}=\ldots=x_{n}\right\}$. We prove the Artin approximation property for them, but we do not touch the approximation "to the smallest term" (see [Z4]) since it is more analytic in nature.

We give a Gevrey version of the Weierstrass-Hironaka division theorem: the division by a finite family $f_{1}, \ldots, f_{r}$ of Gevrey's series. In general, when the data are of Gevrey's order $s$, the quotient and the remainder are not of the same order, as we can see in the following counter-example.

Let $g=\sum_{n, m=1}^{\infty} n ! m ! x^{n} y^{m}$ and $f=-y^{2}-\sum_{n=1}^{\infty} x^{n}$. Then $g=q f+r$, where $q=$ $\sum_{\alpha, \beta \in \mathbb{N}} q_{\alpha \beta} x^{\alpha} y^{\beta}$ is the solution of the infinite system

$$
\begin{aligned}
& q_{0 \beta}=(\beta+2) ! \\
& q_{\alpha \beta}=\alpha !(\beta+2) !+\sum_{\sigma=0}^{\alpha-1} q_{\sigma, \beta+2}, \quad \alpha \geqslant 1 .
\end{aligned}
$$

The Gevrey order of $q$ is at least $(2,1)$, but $f$ and $g$ are Gevrey's series of order $(1,1)$. Nevertheless there are some special cases where you can divide without increasing the Gevrey order (see Remark 4.3). The Gevrey order of the quotients and the remainder we give here improve the result obtained by the author in her Ph.D. Thesis ([Z3], 1994).

The Weierstrass division theorem has also been studied by Chaumat and Chollet ([CC1]). They only considered the case of the division by one Gevrey's series. They gave the preparation theorem in ultradifferentiable classes in [CC2].

1. Preliminaries. Denote by $\mathbb{K}$ the field of real numbers, $\mathbb{R}$, or the field of complex numbers, $\mathbb{C}$. Let $n$ be a positive integer greater than one. We will use the following multiindex notation.

Let $\alpha=\left(\alpha_{1}, \ldots, \alpha_{n}\right) \in \mathbb{N}^{n}$, then

$$
|\alpha|=\alpha_{1}+\ldots+\alpha_{n} .
$$

We put $x^{\alpha}=x_{1}^{\alpha_{1}} \cdots x_{n}^{\alpha_{n}}$ with $x=\left(x_{1}, \ldots, x_{n}\right) \in \mathbb{K}^{n}$, and if $s=\left(s_{1}, \ldots, s_{n}\right) \in[0, \infty)^{n}$,

$$
\alpha !^{s}=\alpha_{1} !^{s_{1}} \cdots \alpha_{n} !^{s_{n}} .
$$

If $\ell \in[0, \infty)$ we put $\underline{\ell}=(\ell, \ldots, \ell)$. Now, for $\alpha=\left(\alpha_{1}, \ldots, \alpha_{n}\right)$ and $\beta=\left(\beta_{1}, \ldots, \beta_{n}\right)$ in $[0, \infty)^{n}$, we put $\alpha \leqslant \beta$ (resp. $\alpha<\beta$ ) if and only if $\beta_{j}-\alpha_{j}$ is a non-negative (resp. positive) real number for each $j=1, \ldots, n$.

Let $\underline{X}=\left(X_{1}, \ldots, X_{n}\right)$ be a vector of independent variables over $\mathbb{K}$, and $f=\sum f_{\alpha} \underline{X}^{\alpha}$ a formal power series over $\mathbb{K}$, that is, an element of the ring $\mathbb{K}[[\underline{X}]]$. We denote by $\operatorname{ord}_{0}(f)$ the integer

$$
\operatorname{ord}_{0}(f)=\min \left\{|\alpha|: f_{\alpha} \neq 0\right\} .
$$

The ring of convergent power series over $\mathbb{K}$ (in the variables $\underline{X}$ ) is denoted by $\mathbb{K}\{\underline{X}\}$. Moreover, whenever we have a polynomial in a new variable $Z$ with coefficients in a power series ring (e.g. $F \in \mathbb{K}[[\underline{X}]][Z]$ ), we emphasize this fact using the ";" sign (e.g. $F(\underline{X} ; Z)$ ). For a square matrix $A, \kappa(A ; z)$ is its characteristic polynomial in the variable $z$. The ring of $p \times q$ matrices with entries in a given ring $R$ is denoted by $\mathbb{M}_{p, q}(R)$. If $P(z)$ and $Q(z)$ are two polynomials with coefficients in $R$, then $\operatorname{Res}_{z}(P, Q)$ is the resultant of $P$ and $Q$. 
The set $\mathbb{K}[[\underline{X}]]_{s}$ is the subring of $\mathbb{K}[[\underline{X}]]$ defined as follows:

$$
f=\sum_{\alpha \in \mathbb{N}^{n}} f_{\alpha} \underline{X}^{\alpha} \in \mathbb{K}[[\underline{X}]]_{s} \Longleftrightarrow f=\sum_{\alpha \in \mathbb{N}^{n}} \frac{f_{\alpha}}{\alpha !^{s}} \underline{X}^{\alpha} \in \mathbb{K}\{\underline{X}\} .
$$

This ring is called the Gevrey formal power series ring of order s. An element of this ring is a Gevrey formal power series of order s. A formal power series $f \in \mathbb{K}[[\underline{X}]]$ is called of Gevrey type if there exists an $s \in[0, \infty)^{n}$ such that $f \in \mathbb{K}[[\underline{X}]]_{s}$. Then the vector $s$ is called its Gevrey order. Observe that whenever $s \leqslant t$ we have

$$
\mathbb{K}[[\underline{X}]]_{s} \subset \mathbb{K}[[\underline{X}]]_{t}
$$

Hence, you have a filtration indexed by the semigroup $\mathcal{S}=[0, \infty)^{n}$. Therefore, we see that the convergent power series are in $\bigcap_{s \in \mathcal{S} \backslash\{0\}} \mathbb{K}[[\underline{X}]]_{s}$, but these rings are not equal. For instance, when $n=1$, the series $\sum m !^{1 / \ln (\ln m)} X^{m}$ is not convergent, but belongs to $\bigcap_{s>0} \mathbb{K}[[X]]_{s}$. Let

$$
\mathbb{K}[[\underline{X}]]_{s,+}=\bigcap_{r>s} \mathbb{K}[[\underline{X}]]_{r}
$$

If $W_{1}, \ldots, W_{d}$ are independent variables over $\mathbb{K}$ (distinct from the $X_{i}$ ), and $t \in[0, \infty)^{d}$, we put

$$
\left(\mathbb{K}[[\underline{W}]]_{t}\right)[[\underline{X}]]_{s,+}=\bigcap_{r>s} \mathbb{K}[[\underline{W}, \underline{X}]]_{(t, r)}
$$

for $s$ in $[0, \infty)^{n}$.

Let $s=\left(s_{1}, \ldots, s_{n}\right) \in[0, \infty)^{n}$ and $\rho \in(0, \infty)$. Define

$$
\|f\|_{s, \rho}=\sum_{\alpha \in \mathbb{N}^{n}} \frac{\left|f_{\alpha}\right|}{\alpha !^{s}} \rho^{\alpha} \quad \text { for } f=\sum_{\alpha \in \mathbb{N}^{n}} f_{\alpha} \underline{X}^{\alpha} \in \mathbb{K}[[\underline{X}]],
$$

where $\rho^{\alpha}=\rho^{|\alpha|}$, and

$$
E_{\rho}^{(s)}=\left\{f \in \mathbb{K}[[\underline{X}]]:\|f\|_{s, \rho} \text { is finite }\right\}
$$

It is easy to see that $\|\cdot\|_{s, \rho}$ is a norm. The $\mathbb{K}$-algebras $E_{\rho}^{(s)}$ are Banach algebras. Moreover, if $\rho^{\prime}>\rho$ then $E_{\rho^{\prime}}^{(s)} \subset E_{\rho}^{(s)}$. Hence the inductive limit

$$
G^{(s)}=\lim _{\rho \rightarrow \infty} E_{\rho}^{(s)}
$$

is a complete topological vector space with the inductive limit topology. Then we have morphisms (of topological vector spaces) $i_{\rho}: E_{\rho}^{(s)} \longrightarrow G^{(s)}$ for each $\rho>0$. The algebra $G^{(s)}$ is a pseudo-Banach algebra whose bound structure is given by the unit balls of the algebras $E_{\rho}^{(s)}$ (see $\left.[\mathrm{ADM}]\right)$. Hence, we obtain $\left({ }^{1}\right)$

Proposition 1.1. The space $G^{(s)}$ is isomorphic to $\mathbb{K}[[\underline{X}]]_{s}$ as $\mathbb{K}$-algebras, but not as topological vector spaces.

$\left({ }^{1}\right)$ Because $\mathbb{K}[[\underline{X}]]_{s}$ is not complete for the $\left(X_{1}, \ldots, X_{n}\right)$-adic topology. 
2. The formal Borel transform in Gevrey's series rings. Fix $n \in \mathbb{N}, n \geqslant 1$, and $s=\left(s_{1}, \ldots, s_{n}\right) \in[0, \infty)^{n}$. Given a Gevrey series

$$
f(\underline{X})=\sum_{\alpha \in \mathbb{N}^{n}} f_{\alpha} \underline{X}^{\alpha} \in G^{(s)} \text {, with } f(\underline{0})=0,
$$

we define its formal Borel transform of level $s$ by the formula

$$
\widehat{\mathcal{B}}_{s} f(\underline{\xi})=\sum_{\alpha \in \mathbb{N}^{n}} \frac{f_{\alpha}}{\alpha !^{s}} \underline{\xi}^{\alpha} .
$$

For the unit 1 of $G^{(s)}$ we put $\widehat{\mathcal{B}}_{s} 1=\delta$, where $\delta$ is the Dirac distribution. Then we have defined a map (of vector spaces over $\mathbb{K}$ ) $\widehat{\mathcal{B}}_{s}: G^{(s)} \longrightarrow \mathbb{K}\{\underline{X}\}$ that is surjective. We put

$$
\left|\widehat{\mathcal{B}}_{s}\right| f(\underline{\xi})=\sum_{\alpha \in \mathbb{N}^{n}} \frac{\left|f_{\alpha}\right|}{\alpha !^{s} \underline{\xi}^{\alpha}} .
$$

and call it the absolute Borel transform of level s.

Property 2.1 (the evaluation property). Let $\underline{Y}=\left(Y_{1}, \ldots, Y_{n}\right), \underline{X}=\left(X_{1}, \ldots, X_{m}\right)$ be variables over $\mathbb{K}$, and $s=\left(s_{1}, \ldots, s_{n}\right) \in[0, \infty)^{n}, \tau_{j}=\left(\tau_{j 1}, \ldots, \tau_{j m}\right) \in[0, \infty)^{m}$ for $j=1, \ldots, n$. Let $s *=\max \left\{s_{1}, \ldots, s_{n}\right\}$. If $F(\underline{Y})$ is in the ring $\mathbb{K}[[\underline{Y}]]_{s}$ and $f_{j}(\underline{X})$ in $\mathbb{K}[[\underline{X}]]_{\tau_{j}}$, then, whenever $f_{1}(\underline{0})=\ldots=f_{n}(\underline{0})=0$, the composition $F \circ\left(f_{1}, \ldots, f_{n}\right)$ is in $\mathbb{K}[[\underline{X}]]_{r}$ for $r=\left(r_{1}, \ldots, r_{m}\right)$ with $r_{j}=\max \left\{\tau_{1 j}, \ldots, \tau_{n j}, s *\right\}$.

Proof. Let $\delta>0$ be such that the series $\left|\widehat{\mathcal{B}}_{\tau_{j}}\right| f_{j}$ are convergent in $(-\delta, \delta) \times \ldots \times$ $(-\delta, \delta)$. Take $\epsilon>0$ such that $\left|\widehat{\mathcal{B}}_{s}\right| F$ is convergent in $(-\epsilon, \epsilon) \times \cdots \times(-\epsilon, \epsilon)$. The analytic functions

$$
\varphi_{j}:(0, \delta) \ni x \longmapsto\left|\widehat{\mathcal{B}}_{\tau_{j}}\right| f_{j}(x, \ldots, x)=\left\|f_{j}\right\|_{\tau_{j}, x} \in \mathbb{R}
$$

can be factorized as $\varphi_{j}(x)=x \beta_{j}(x)$ in $(0, \delta)$, where the $\beta_{j}$ are positive increasing real analytic functions.

Choose $\delta^{\prime}<\delta$ and put $K_{j}=\beta_{j}\left(\delta^{\prime}\right), 1 \leqslant j \leqslant n$. Taking $\delta_{1}<\delta^{\prime}$ such that $\delta_{1} K_{j}<\epsilon$ for $j=1, \ldots, n$, we obtain the inequalities

$$
\varphi_{j}(x)<\epsilon \text { in }\left(0, \delta_{1}\right)
$$

Hence $\left(\left|\widehat{\mathcal{B}}_{s}\right| F\right)\left(\varphi_{1}(x), \ldots, \varphi_{n}(x)\right)$ is finite for $x \in\left(0, \delta_{1}\right)$.

Let $r \in[0, \infty)^{m}$ be as in the statement. Assume the inequality

$$
\left|\widehat{\mathcal{B}}_{r}\right|\left(F \circ\left(f_{1}, \ldots, f_{n}\right)\right) \leqslant\left|\widehat{\mathcal{B}}_{s} F\right| \circ\left(\left|\widehat{\mathcal{B}}_{\tau_{1}}\right| f_{1}, \ldots,\left|\widehat{\mathcal{B}}_{\tau_{n}}\right| f_{n}\right)
$$

in the interval $\mathcal{Q}=\left(0, \delta_{1}\right) \times{ }^{m} . \times\left(0, \delta_{1}\right)$. Then, if $x \in \mathcal{Q} \cap\left\{x_{1}=\ldots=x_{n}\right\}$, we get

$$
\left\|F \circ\left(f_{1}, \ldots, f_{n}\right)\right\|_{r, x} \leqslant\left(\left|\widehat{\mathcal{B}}_{s}\right| F\right)\left(\varphi_{1}(x), \ldots, \varphi_{n}(x)\right) .
$$

This inequality and (i) imply that $F \circ\left(f_{1}, \ldots, f_{n}\right) \in G^{(r)}$, as was claimed in the statement.

In order to prove (ii), we proceed as follows. Put

$$
F(\underline{Y})=\sum_{i \in \mathbb{N}^{n}} F_{i} \underline{Y}^{i} \quad \text { and } \quad f_{j}(\underline{X})=\sum_{\alpha \in \mathbb{N}^{m}} a_{j \alpha} \underline{X}^{\alpha}, \quad 1 \leqslant j \leqslant n .
$$


For $t=0,1, \ldots$, and $j=1,2, \ldots, n$, we have the formal power series expansion

$$
\left(f_{j}\right)^{t}=\sum_{\beta \in \mathbb{N}^{m}} c_{j \beta}^{(t)} \underline{X}^{\beta} \quad \text { where } \quad c_{j \beta}^{(t)}=\sum_{\substack{\left(\alpha_{1}, \ldots, \alpha_{t}\right) \in\left(\mathbb{N}^{m}\right)^{t} \\ \alpha_{1}+\ldots+\alpha_{t}=\beta}} a_{j \alpha_{1}} \cdots a_{j \alpha_{t}} .
$$

Hence, the formal power series

$$
\sum_{i=\left(i_{1}, \ldots, i_{n}\right) \in \mathbb{N}^{n}} F_{i} \sum_{\beta \in \mathbb{N}^{m}}\left(\sum_{\substack{\left(\beta_{1}, \ldots, \beta_{n}\right) \in\left(\mathbb{N}^{m}\right)^{n} \\ \beta_{1}+\ldots+\beta_{n}=\beta}} c_{1 \beta_{1}}^{\left(i_{1}\right)} \cdots c_{n \beta_{n}}^{\left(i_{n}\right)}\right) \underline{X}^{\beta}
$$

is the formal power series expansion of $F \circ\left(f_{1}, \ldots, f_{n}\right)$. Let $s *=\max \left\{s_{1}, \ldots, s_{n}\right\}$ and $r=\left(r_{1}, \ldots, r_{m}\right) \in[0, \infty)^{m}$ be such that $r_{j}=\max \left\{\tau_{1 j}, \ldots, \tau_{n j}, s *\right\}, 1 \leqslant j \leqslant m$. Set

$$
d_{j \beta}^{(t)}=\sum_{\substack{\left(\alpha_{1}, \ldots, \alpha_{t}\right) \in\left(\mathbb{N}^{m}\right)^{t} \\ \alpha_{1}+\ldots+\alpha_{t}=\beta}} \frac{\left|a_{j \alpha_{1}}\right|}{\alpha_{1} !^{\tau_{j}}} \cdots \frac{\left|a_{j \alpha_{t}}\right|}{\alpha_{t} !^{\tau_{j}}}
$$

then for $j=1, \ldots, n$ and $t \in \mathbb{N}$ we have

$$
\left(\left|\widehat{\mathcal{B}}_{\tau_{j}}\right| f_{j}\right)^{t}=\sum_{\beta \in \mathbb{N}^{m}} d_{j \beta}^{(t)} \underline{X}^{\beta}
$$

It is easy to prove the inequalities

$$
\frac{\left|c_{j \beta}^{(t)}\right|}{\beta !^{r}} \leqslant \frac{d_{j \beta}^{(t)}}{t !|r|} \leqslant \frac{d_{j \beta}^{(t)}}{t !^{s^{*}}} .
$$

In consequence,

$$
\begin{aligned}
&\left|\widehat{\mathcal{B}}_{r}\right|\left(F \circ\left(f_{1}, \ldots, f_{n}\right)\right)(\underline{x}) \leqslant \sum_{i \in \mathbb{N}^{n}} \frac{\left|F_{i}\right|}{i !^{s}} \sum_{\beta \in \mathbb{N}^{m}} \sum_{\beta_{1}+\ldots+\beta_{n}=\beta} d_{1 \beta_{1}}^{\left(i_{1}\right)} \cdots d_{n \beta_{n}}^{\left(i_{n}\right)} \underline{x}^{\beta} \\
&=\left|\widehat{\mathcal{B}}_{s}\right| F \circ\left(\left|\widehat{\mathcal{B}}_{\tau_{1}}\right| f_{1}, \ldots,\left|\widehat{\mathcal{B}}_{\tau_{n}}\right| f_{n}\right)(\underline{x})
\end{aligned}
$$

for $\underline{x} \in \mathcal{Q}$.

Remark 2.2. Let $\lambda_{1}, \ldots, \lambda_{n} \in \mathbb{K} \backslash 0$. Then $f\left(Y_{1}, \ldots, Y_{n}\right)$ and $f\left(\lambda_{1} Y_{1}, \ldots, \lambda_{n} Y_{n}\right)$ have the same Gevrey order.

\section{The Hensel lemma for the Gevrey rings}

LEMma 3.1 (the Hensel lemma for the Gevrey rings). Let $f(\underline{X} ; z) \in G^{(s)}[z]$ be a monic polynomial in the variable $z$ such that $f(0 ; z)=a(z) b(z)$ with $\operatorname{gcd}(a(z), b(z))=1$. Then there exist $P(\underline{X} ; z)$ and $Q(\underline{X} ; z)$ in $G^{(s)}[z]$ such that $f=P Q$ and

$$
\begin{aligned}
& P(0 ; z)=a(z), \quad \operatorname{deg}_{z} P=\operatorname{deg}_{z} a=p, \\
& Q(0 ; z)=b(z), \quad \operatorname{deg}_{z} Q=\operatorname{deg}_{z} b=q .
\end{aligned}
$$

It is well known (see $[\mathrm{N}]$, page 104) that there exist $P$ and $Q$ with coefficients in $\mathbb{K}[[\underline{X}]]$ such that $f=P Q$ and

$$
\begin{aligned}
& P(0 ; z)=a(z), \quad \operatorname{deg}_{z} P=\operatorname{deg}_{z} a=p \\
& Q(0 ; z)=b(z), \quad \operatorname{deg}_{z} Q=\operatorname{deg}_{z} b=q .
\end{aligned}
$$


The only difficulty is to prove that the divergent coefficients of $P$ and $Q$ are in fact of Gevrey's order $s$. For that we recall the proof of the Hensel lemma for matrices introduced by P. M. Cohn (see [Co], or [Z2]).

Put

$$
f(\underline{X} ; z)=\mathbf{a}_{0}(\underline{X})+\mathbf{a}_{1}(\underline{X}) z+\ldots+\mathbf{a}_{d-1}(\underline{X}) z^{d-1}+z^{d} .
$$

We can assume that $a(z)=z^{p}$ (because you can argue to induction on $d$ ). The companion matrix of $f$ can be written as

$$
M=\left(\begin{array}{ll}
A_{1} & A_{2} \\
A_{3} & A_{4}
\end{array}\right) \in \mathbb{M}_{d, d}\left(G^{(s)}\right)
$$

where

$$
\begin{gathered}
A_{1}=\left(\begin{array}{ccccc}
0 & 0 & \ldots & 0 & 0 \\
1 & 0 & \ldots & 0 & 0 \\
\vdots & \vdots & \ddots & \vdots & \vdots \\
0 & \ldots & \ldots & 1 & 0
\end{array}\right) \in \mathbb{M}_{p, p}\left(G^{(s)}\right), \quad A_{3}=\left(\begin{array}{cccc}
0 & \ldots & 0 & 1 \\
0 & \ldots & 0 & 0 \\
\vdots & \ldots & \vdots & \vdots \\
0 & \ldots & 0 & 0
\end{array}\right), \\
A_{2}=\left(\begin{array}{cccc}
0 & \ldots & 0 & -\mathbf{a}_{0} \\
\vdots & \ldots & \vdots & \vdots \\
0 & \ldots & 0 & -\mathbf{a}_{p-1}
\end{array}\right), \quad A_{4}=\left(\begin{array}{cccc}
0 & \ldots & 0 & -\mathbf{a}_{p} \\
\vdots & \ldots & \vdots & \vdots \\
0 & \ldots & 0 & -\mathbf{a}_{d-1}
\end{array}\right) \in \mathbb{M}_{q, q}\left(G^{(s)}\right) .
\end{gathered}
$$

We will construct a matrix $X$ such that

$$
\left(\begin{array}{cc}
I & -X \\
0 & I
\end{array}\right) M\left(\begin{array}{cc}
I & X \\
0 & I
\end{array}\right)=\left(\begin{array}{cc}
A_{1}-X A_{3} & 0 \\
A_{3} & A_{3} X+A_{4}
\end{array}\right) .
$$

For that, define a sequence of matrices $X_{r} \in \mathbb{M}_{p, q}(\mathbb{K}[[\underline{X}]])$ :

$$
\left\{\begin{array}{l}
X_{0}=0 \\
X_{r} A_{4}-A_{1} X_{r}=A_{2}-X_{r-1} A_{3} X_{r-1}, \quad r \geqslant 1 .
\end{array}\right.
$$

Now, consider the resultant $u=\operatorname{Res}_{z}\left(\kappa\left(A_{1}, z\right), \kappa\left(A_{4}, z\right)\right)$, then choose two polynomials: $L(z)$ of degree $\leqslant q-1$ and $N(z)$ of degree $\leqslant p-1$, such that $u=L(z) \kappa\left(A_{1}, z\right)+$ $N(z) \kappa\left(A_{4}, z\right)([\mathrm{BR}]$, page 28$)$. The polynomial

$$
H(z)=\frac{1}{u} L(z) \kappa\left(A_{1}, z\right)=\alpha_{p} z^{p}+\ldots+\alpha_{d-1} z^{d-1}
$$

assumes the value 0 at $A_{1}$ and 1 at $A_{4}$. Hence, the matrix

$$
X_{r}=\alpha_{p} C+\sum_{j=p+1}^{d-1} \alpha_{j} \sum_{i+l=j} A_{1}^{i} C A_{4}^{l},
$$

where $C=A_{2}-X_{r-1} A_{3} X_{r-1}$, is the solution of (i). Let $\mathbf{m}$ be the maximal ideal of $\mathbb{K}[[\underline{X}]]$. The limit $X$ of the sequence $\left\{X_{r}\right\}$ - for the $\mathbf{m}$-adic topology in $\mathbb{K}[[\underline{X}]]$ — verifies $(*)$. Then $(-1)^{d} f$ is the product of the characteristic polynomials of $A_{1}-X A_{3}$ and $A_{3} X+A_{4}$.

In order to prove that the coefficients of $X$ are in some $E_{\epsilon}^{(s)}$ we will use the following result. 
Lemma 3.2. Let $\underline{X}, y, z$ be independent variables over the field $\mathbb{K}$, and $p, q \in \mathbb{N}$ be such that $p q \neq 0$. Denote by $\mathbb{F}$ the fraction field of the polynomial ring $\mathbb{K}[y]$. Set $R=\mathbb{F}[[\underline{X}]]$. Consider the monic polynomials

$$
\begin{aligned}
& P(z)=c_{0}+c_{1} z+\ldots+c_{q-1} z^{q-1}+z^{q} \in \mathbb{K}[[\underline{X}]][z], \\
& \widetilde{P}(z)=\frac{c_{0}}{y^{q}}+\frac{c_{1}}{y^{q-1}} z+\ldots+\frac{c_{q-1}}{y} z^{q-1}+z^{q} \in R[z]
\end{aligned}
$$

with $c_{0} \neq 0$. Let $L, N \in \mathbb{K}[[\underline{X}]][z]$ (resp. $\left.\widetilde{L}, \widetilde{N} \in R[z]\right)$ be such that $\operatorname{Res}_{z}\left(z^{p}, P(z)\right)=$ $L(z) z^{p}+N(z) P(z)\left(\operatorname{resp} \cdot \operatorname{Res}_{z}\left(z^{p}, \widetilde{P}(z)\right)=\widetilde{L}(z) z^{p}+\widetilde{N}(z) \widetilde{P}(z)\right)$ and $\operatorname{deg}_{z} L \leqslant q-1$, $\operatorname{deg}_{z} N \leqslant p-1\left(\right.$ resp. $\left.\operatorname{deg}_{z} \widetilde{L} \leqslant q-1, \operatorname{deg}_{z} \widetilde{N} \leqslant p-1\right)$. Set

$$
\begin{aligned}
& L(z)=\nu_{0}+\nu_{1} z+\ldots+\nu_{q-1} z^{q-1} \in \mathbb{K}[[\underline{X}]][z], \\
& \widetilde{L}(z)=\widetilde{\nu}_{0}+\widetilde{\nu}_{1} z+\ldots+\widetilde{\nu}_{q-1} z^{q-1} \in R[z] .
\end{aligned}
$$

Then $\widetilde{\nu}_{j}=\nu_{j} y^{j+p-p q}$ for $j=0,1, \ldots, q-1$.

Proof. Consider the extension of the identity over $R$ to the polynomial ring $R[z]$ given by $\pi(z)=y z$. Then

$$
\operatorname{Res}_{z}\left(z^{p}, P\right)=\pi(L) y^{p} z^{p}+\pi(N) y^{q} \widetilde{P}(z) .
$$

Since $\left({ }^{2}\right) \operatorname{Res}_{z}\left(z^{p}, P\right)=y^{p q} \operatorname{Res}_{z}\left(z^{p}, \widetilde{P}\right)$, we have

$$
\operatorname{Res}_{z}\left(z^{p}, P\right)=y^{p q} \widetilde{L}(z) z^{p}+y^{p q} \widetilde{N}(z) \widetilde{P}(z) .
$$

Consequently, $\widetilde{L}=y^{p-p q} \pi(L)$, and that implies the statement.

Now consider $\rho \in(0,1)$ such that the resultant $u$ is a unit in $E_{\rho}^{(s)}$ and the coefficients of $L$ and $N$ are in $E_{\rho}^{(s)}$. Fix $a_{0}, \ldots, a_{d-1}$ in $E_{\rho}^{(s)}$ such that $i_{\rho}\left(a_{j}\right)=\mathbf{a}_{j}$ (if it is impossible, take a smaller radius $\rho$ at the beginning).

Take the real analytic functions

$$
\begin{aligned}
\varphi_{j}(x) & =\left|\widehat{\mathcal{B}}_{s}\right| a_{j}(x, \ldots, x), & & 0 \leqslant j \leqslant d-1, \\
\xi_{\ell}(x) & =\left|\widehat{\mathcal{B}}_{s}\right| \alpha_{\ell}(x, \ldots, x), & & p \leqslant \ell \leqslant d-1 .
\end{aligned}
$$

Let $\rho^{\prime} \in(0, \rho)$. For each $j \in\{0, \ldots, p-1\}$ the function $\varphi_{j}$ can be factorized as $\varphi_{j}(x)=$ $x \beta_{j}(x)$ in $\left[-\rho^{\prime}, \rho^{\prime}\right]$, where $\beta_{j}$ is a positive increasing real analytic function. Let

$$
\tau=\max \left\{\xi_{\ell}\left(\rho^{\prime}\right): 1 \leqslant \ell \leqslant t\right\} \cup\left\{\varphi_{j}\left(\rho^{\prime}\right): p \leqslant j \leqslant d-1\right\}>0 .
$$

Let $y$ be a new variable over $\mathbb{K}$. Consider the monic polynomial

$$
g_{y}(\underline{X} ; z)=\frac{1}{y^{d}} f(\underline{X} ; y z) \in R[z],
$$

where $R$ is as in Lemma 3.2. The algorithm (i) can be performed for $g_{y}$. We have now

$$
A_{2}^{\prime}=\left(\begin{array}{cccc}
0 & \ldots & 0 & -\mathbf{a}_{0} / y^{d} \\
\vdots & \ldots & \vdots & \vdots \\
0 & \ldots & 0 & -\mathbf{a}_{p-1} / y^{d-p+1}
\end{array}\right), \quad A_{4}^{\prime}=\left(\begin{array}{cccc}
0 & \ldots & 0 & -\mathbf{a}_{p} / y^{d-p} \\
\vdots & \ldots & \vdots & \vdots \\
0 & \ldots & 0 & -\mathbf{a}_{d-1} / y
\end{array}\right),
$$

$\left({ }^{2}\right)$ See $[\mathrm{BR}]$, page 29. 
and $A_{1}, A_{3}$ as before. Then there exist two new polynomials $\widetilde{L}_{y}$ and $\widetilde{N} \in R[z]$ such that $\widetilde{u}=\operatorname{Res}_{z}\left(\kappa\left(A_{1}, z\right), \kappa\left(A_{4}^{\prime}, z\right)\right)=\widetilde{L}(z) \kappa\left(A_{1}, z\right)+\widetilde{N}(z) \kappa\left(A_{4}^{\prime}, z\right)$ and $\operatorname{deg}_{z} \widetilde{L}_{y} \leqslant q-1$, $\operatorname{deg}_{z} \tilde{N} \leqslant p-1$. Set

$$
\widetilde{H}_{y}(z)=\frac{1}{\widetilde{u}} \widetilde{L}_{y}(z) \kappa\left(A_{1}, z\right)=\widetilde{\alpha}_{p}(\underline{X}, y) z^{p}+\ldots+\widetilde{\alpha}_{d-1}(\underline{X}, y) z^{d-1} .
$$

Lemma 3.2 for $P(z)=\kappa\left(A_{4}, z\right)$ and $\widetilde{P}(z)=\kappa\left(A_{4}^{\prime}, z\right)$ implies the equalities

$$
\widetilde{\alpha}_{j}(\underline{X}, y)=\frac{\widetilde{\nu}_{j-p}}{\widetilde{u}}=y^{j} \frac{\nu_{j-p}}{u}=y^{j} \alpha_{j}(\underline{X}), \quad p \leqslant j \leqslant d-1,
$$

since $u=y^{p q} \widetilde{u}$. The sequence of matrices $\widetilde{X}_{r}(y)$ given inductively by the iteration

$$
\begin{aligned}
\widetilde{X}_{0}(y) & =0 \\
\widetilde{X}_{r}(y) A_{4}^{\prime}-A_{1} \widetilde{X}_{r}(y) & =A_{2}^{\prime}-\widetilde{X}_{r-1}(y) A_{3} \widetilde{X}_{r-1}(y), \quad r \geqslant 1,
\end{aligned}
$$

is the sequence

$$
\widetilde{X}_{r}(y)=\widetilde{\alpha}_{p}(\underline{X}, y) \widetilde{C}+\sum_{j=p+1}^{d-1} \widetilde{\alpha}_{j}(\underline{X}, y) \sum_{i+l=j} A_{1}^{i} \widetilde{C}\left(A_{4}^{\prime}\right)^{l},
$$

where $\widetilde{C}=A_{2}^{\prime}-\widetilde{X}_{r-1}(y) A_{3} \widetilde{X}_{r-1}(y)$. Now consider $\widetilde{Z}_{r}(y)=\widetilde{X}_{r}(y)-\widetilde{X}_{r-1}(y)$. Then we have the equalities

$$
\widetilde{Z}_{r}(y) A_{4}^{\prime}-A_{1} \widetilde{Z}_{r}(y)=\widetilde{D}
$$

and

$$
\widetilde{Z}_{r}(y)=\widetilde{\alpha}_{p}(\underline{X}, y) \widetilde{D}+\sum_{j=p+1}^{d-1} \widetilde{\alpha}_{j}(\underline{X}, y) \sum_{i+l=j} A_{1}^{i} \widetilde{D}\left(A_{4}^{\prime}\right)^{l},
$$

where $\widetilde{D}=-\widetilde{X}_{r-1}(y) A_{3} \widetilde{Z}_{r-1}(y)-\widetilde{Z}_{r}(y) A_{3} \widetilde{X}_{r-1}(y)$. Set

$$
w(t)=1+\sum_{j=p+1}^{d-1} \sum_{l=0}^{j}(\tau / t)^{l} \quad \text { for } t \in(0, \infty) .
$$

Let $\lambda \in(1, \infty)$ be such that $W=\tau \lambda^{d-1} w(\lambda)>e$. Now, put $y=\lambda$ in the previous construction, that is, consider:

$$
\begin{gathered}
g(\underline{X}, z)=g_{\lambda}(\underline{X}, z), \quad \widetilde{\alpha}_{j}=\widetilde{\alpha}_{j}(\underline{X}, \lambda), \quad j=p, \ldots, d-1, \\
\widetilde{H}(z)=\widetilde{H}_{\lambda}(z), \quad \widetilde{X}_{r}=\widetilde{X}_{r}(\lambda), \quad \widetilde{Z}_{r}=\widetilde{Z}_{r}(\lambda) .
\end{gathered}
$$

Let us take the polynomial with analytic coefficients

$$
\Gamma(t, z)=\left\|\widetilde{\alpha}_{p}\right\|_{s, t}+\sum_{j=p+1}^{d-1}\left\|\widetilde{\alpha}_{j}\right\|_{s, t} \sum_{l=0}^{j} z^{l} \text { for } t \in\left(0, \rho^{\prime}\right) .
$$

Let $\widetilde{W}>W$ be such that $4 \widetilde{W}^{3}<\exp (\widetilde{W})$. Take $\rho^{\prime \prime} \in\left(0, \rho^{\prime}\right)$ and put

$$
c=\max \left\{\beta_{0}\left(\rho^{\prime \prime}\right) \lambda^{-d}, \ldots, \beta_{p-1}\left(\rho^{\prime \prime}\right) \lambda^{-d+p-1}\right\} .
$$

Now choose $\epsilon \in\left(0, \rho^{\prime \prime}\right)$ such that $\epsilon \widetilde{W} \exp (\widetilde{W})<1$ and $\delta \in\left(0, \rho^{\prime \prime}\right)$ with $\delta c<\epsilon$. Then $\left\|A_{2}^{\prime}\right\|_{s, \delta} \leqslant \delta c<\epsilon$. 
The iteration

$$
\begin{aligned}
& u_{0}=0 \\
& u_{r}=I\left(u_{r-1}\right), \quad r \geqslant 1,
\end{aligned}
$$

for $I(t)=\widetilde{W}\left(\epsilon+x^{2}\right)$, gives us a sequence of non-negative real numbers $\left\{u_{r}\right\}$ such that

$$
\left\|\widetilde{X}_{r}\right\|_{s, \delta} \leqslant u_{r} \leqslant \xi_{0}
$$

where $\xi_{0}$ satisfies $I\left(\xi_{0}\right)=\xi_{0}$ and $1 /\left(2 \widetilde{W}^{2}\right)>\xi_{0}$. Therefore

So, in particular

$$
\left\|\widetilde{Z}_{r}\right\|_{s, \delta} \leqslant \frac{1}{\widetilde{W}}\left\|\widetilde{Z}_{r-1}\right\|_{s, \delta}
$$

$$
\lim _{r \longrightarrow \infty}\left\|\widetilde{Z}_{r}\right\|_{s, \delta}=0 .
$$

The sequence $\left\{\widetilde{X}_{r}\right\}$ has the limit $\sum_{r=1}^{\infty} \widetilde{Z}_{r}$ in the ring of $p \times q$ matrices with entries in $E_{\delta}^{(s)}$, therefore, also in $\mathbb{M}_{p, q}\left(G^{(s)}\right)$. In consequence $g$ can be factorized as $g(\underline{X} ; z)=$ $G_{1}(\underline{X} ; z) G_{2}(\underline{X} ; z)$ where $G_{j} \in G^{(s)}[z]$ and $j=1,2$. Then

$$
P(\underline{X} ; z)=\lambda^{p} G_{1}(\underline{X} ; z / \lambda) \text { and } Q(\underline{X} ; z)=\lambda^{q} G_{2}(\underline{X} ; z / \lambda)
$$

are in $G^{(s)}[z]$, as we have stated in Lemma 3.1 .

4. The division theorems. Let $Z=\left(Z_{1}, \ldots, Z_{c}\right)$ and $W=\left(W_{1}, \ldots, W_{d}\right)$ be two vectors of variables over $\mathbb{K}$. Given a formal power series

$$
f(W, Z)=\sum_{A \in \mathbb{N}^{c}} f_{A}(W) Z^{A}=\sum_{(A, B) \in \mathbb{N}^{c} \times \mathbb{N}^{d}} f_{A B} W^{B} Z^{A},
$$

we define the sets of exponents

$$
\begin{aligned}
\operatorname{Exp}(f) & =\left\{(A, B) \in \mathbb{N}^{c} \times \mathbb{N}^{d}: f_{A B} \neq 0\right\}, \\
\operatorname{Exp}_{Z}(f) & =\left\{A \in \mathbb{N}^{c}: f_{A}(W) \neq 0\right\} .
\end{aligned}
$$

In order to prove the Weierstrass-Hironaka theorem we will use the following lemma.

LEMmA 4.1. Let $a>0, h>0$ and $\ell \geqslant 1$. The function

$$
\varphi_{\ell, a, h}:[0, \infty) \ni x \longmapsto \frac{(x+\ell)^{a}}{h^{x}} \in \mathbb{R}
$$

has an absolute maximum $m_{h}$ such that $\varphi_{\ell, a, h}\left(m_{h}\right) \geqslant 1$. Moreover, when $h>h^{\prime}$ we have

$$
\varphi_{\ell, a, h^{\prime}}\left(m_{h}\right) \leqslant \frac{Q_{\ell, a, h^{\prime}}}{h^{a}}
$$

where $Q_{\ell, a, h^{\prime}}$ is a constant depending only on $\ell, a$ and $h^{\prime}$.

Let $t=\left(t_{1}, \ldots, t_{d}\right) \in[0, \infty)^{d}$ and $s=\left(s_{1}, \ldots, s_{c}\right) \in[0, \infty)^{c}$. We put $(t, s)=$ $\left(t_{1}, \ldots, t_{d}, s_{1}, \ldots, s_{c}\right) \in[0, \infty)^{c+d}$. If $\mu=\left(\mu_{1}, \ldots, \mu_{c}\right) \in[0, \infty)^{c}$, then $\langle\mu, s\rangle=\sum_{j=1}^{c} \mu_{j} s_{j}$.

TheOREM 4.2 (the Weierstrass-Hironaka division theorem for Gevrey's rings). Let $r \in \mathbb{N} \backslash 0$ and $A_{0}=(0, \ldots, 0), A_{1}, \ldots, A_{r} \in \mathbb{N}^{c}$. Consider some non-units $f_{1}, \ldots, f_{r} \in$ $\mathbb{K}[[W, Z]]_{(t, s)}$ such that for any $k \in\{1, \ldots, r\}$ we have

(a) The pair $\left(A_{k}, 0\right)$ is an element of $\operatorname{Exp}\left(f_{k}\right)$.

(b) If $(U, 0) \in \operatorname{Exp}\left(f_{k}\right)$ then $|U|>\left|A_{k}\right|$. 
Take a finite partition $\left\{\mathcal{P}_{1}, \ldots, \mathcal{P}_{r}\right\}$ of $\mathbb{N}^{c}$, such that $\mathcal{P}_{k} \subset A_{k}+\mathbb{N}^{c}$, and $A_{k} \in \mathcal{P}_{k}$ or $\mathcal{P}_{k}=\emptyset$ for $k=1, \ldots, r$. If $A_{i q}$ is the $q$-th component of $A_{i}$, we define

$$
\mu_{q}=\max \left\{A_{i q}: 1 \leqslant i \leqslant r\right\}
$$

where $q \in\{1, \ldots, c\}$, and $\mu=\left(\mu_{1}, \ldots, \mu_{c}\right) \in \mathbb{N}^{c}$. Let $t^{\prime}=\left(t_{1}+\langle\mu, s\rangle, \ldots, t_{d}+\langle\mu, s\rangle\right)$ and $s^{\prime}=(|s|, \ldots,|s|) \in[0, \infty)^{c}$. Then for each $g \in \mathbb{K}\left[[W, Z]_{(t, s)}\right.$ there exist $h_{0}, h_{1}, \ldots, h_{r}$ in $\mathbb{K}[[W, Z]]_{\left(t^{\prime}, s^{\prime}\right)}$ such that

(1) $g=h_{1} f_{1}+\ldots+h_{r} f_{r}+h_{0}$;

(2) $\operatorname{Exp}_{Z}\left(h_{k} Z^{A_{k}}\right) \subset \mathcal{P}_{k}, 0 \leqslant k \leqslant r$;

(3) $\operatorname{ord}_{0}\left(h_{k}\right) \geqslant \operatorname{ord}_{0}(g)-\operatorname{ord}_{0}\left(f_{k}\right), 1 \leqslant k \leqslant r$.

Moreover, the $h_{j}$ 's are the unique formal power series satisfying (1), (2) and (3).

Proof. Write

$$
\begin{gathered}
f_{k}=\sum_{(U, V) \in \mathbb{N}^{c} \times \mathbb{N}^{d}} f_{k, U, V} W^{V} Z^{U} \quad \text { where } f_{k, U, V} \in \mathbb{K}, \\
g=\sum_{(U, V) \in \mathbb{N}^{c} \times \mathbb{N}^{d}} g_{U V} W^{V} Z^{U} .
\end{gathered}
$$

We can suppose that $f_{k, A_{k}, 0}=1$ and $g, f_{k}$ are in $E_{1 / \rho}^{(t, s)}$ for some $\rho<1$ and all $k \in\{1, \ldots, r\}$ (by Remark 2.2). Therefore, there exists $\delta>0$ such that

$$
\frac{\left|g_{U V}\right|}{U !^{s} V !^{t}} \leqslant \delta \rho^{|U|+|V|} \text { and } \frac{\left|f_{k U V}\right|}{U !^{s} V !^{t}} \leqslant \delta \rho^{|U|+|V|}
$$

for $k=1, \ldots, r$ and $(U, V) \in \mathbb{N}^{c} \times \mathbb{N}^{d}$. It is well known (see [AHV]) that there exist $r+1$ elements $h_{0}, h_{1}, \ldots, h_{r}$ in the formal power series ring $\mathbb{K}[[W, Z]]$ satisfying (1), (2) and (3). We can construct them as follows. Put

$$
P_{k}=f_{k}-Z^{A_{k}}=\sum_{(A, B) \in \mathbb{N}^{c} \times \mathbb{N}^{d}} F_{k, A, B} W^{B} Z^{A} .
$$

Then we expand $g$ as the finite sum:

$$
g=\sum_{k=1}^{r} h_{k}^{(0)} Z^{A_{k}}+h_{0}^{(0)}=\sum_{k=1}^{r} f_{k} h_{k}^{(0)}+h_{0}^{(0)}-\sum_{k=1}^{r} P_{k} h_{k}^{(0)},
$$

where $\operatorname{Exp}_{Z}\left(h_{k}^{(0)} Z^{A_{k}}\right) \subset \mathcal{P}_{k}$. Analogously, we write

$$
-\sum_{k=1}^{r} P_{k} h_{k}^{(0)}=\sum_{k=1}^{r} h_{k}^{(1)} Z^{A_{k}}+h_{0}^{(1)}=\sum_{k=1}^{r} f_{k} h_{k}^{(1)}+h_{0}^{(1)}-\sum_{k=1}^{r} P_{k} h_{k}^{(1)},
$$

where $\operatorname{Exp}_{Z}\left(h_{k}^{(1)} Z^{A_{k}}\right) \subset \mathcal{P}_{k}$. Consequently,

$$
g=\sum_{i=1}^{r} f_{k}\left(h_{k}^{(0)}+h_{k}^{(1)}\right)+\left(h_{0}^{(0)}+h_{0}^{(1)}\right)-\sum_{k=1}^{r} P_{k} h_{k}^{(1)} .
$$

This algorithm gives us the required formal power series

$$
h_{k}=\sum_{j=0}^{\infty} h_{k}^{(j)}, \quad 0 \leqslant k \leqslant r
$$


and the order conditions
$\left(\nu_{k j}\right)$
$\operatorname{ord}_{0}\left(h_{k}^{(j)} z^{A_{k}}\right) \geqslant\left|A_{k}\right|+j$

(see $[\mathrm{AHV}]$ for the details). Now, each $h_{k}^{(j)}$ can be expanded as

$$
h_{k}^{(j)}=\sum_{(A, B) \in \mathbb{N}^{c} \times \mathbb{N}^{d}} H_{k A B}^{(j)} W^{B} Z^{A} \quad \text { for some } H_{k A B}^{(j)} \in \mathbb{K} .
$$

Moreover, we have the inductive formula.

For $j=0$ and $k=0, \ldots, r$,

$$
H_{k A B}^{(0)}= \begin{cases}g_{A+A_{k}, B} & \text { if } A+A_{k} \in \mathcal{P}_{k} \\ 0 & \text { otherwise. }\end{cases}
$$

For $j>0$ and $k=0, \ldots, r$,

$$
H_{k A B}^{(j)}= \begin{cases}-\sum_{i=1}^{r} \sum_{\substack{U_{1}+U_{2}=A+A_{k} \\ V_{1}+V_{2}=D}} F_{i U_{1} V_{1}} H_{i U_{2} V_{2}}^{(j-1)} & \text { if } A+A_{k} \in \mathcal{P}_{k} \\ 0 & \text { otherwise. }\end{cases}
$$

If $N=\max \left\{A_{i q}: 0 \leqslant i \leqslant r, 1 \leqslant q \leqslant c\right\}$, then we choose

(1) a real number $K$ satisfying $K \geqslant 2 r \delta \prod_{i=1}^{r} A_{i} !^{s} /(1-\rho)^{c}$ and greater than each of the numbers $2,2 \max \left\{\left|g_{A_{k}, 0}\right|: k=1, \ldots, r\right\}$ and

$$
\delta \prod_{\left\{(i, q): s_{q} A_{i q} \neq 0\right\}} \varphi_{i q}\left(m_{i q}\right),
$$

where $\varphi_{i q}$ is the function from Lemma 4.1 for $a=s_{q} A_{i q}, \ell=A_{i q}$ and $h=N$, and $m_{i q}$ is its maximum;

(2) a real number $T$ greater than $\max \left\{N,(r \delta / 2)^{1 / c},\left(2 r \delta Q^{\prime} /(1-\rho)^{c}\right)^{1 / \sigma}\right\}$, where $\sigma=$ $\min \left\{\left\langle A_{1}, s\right\rangle, \ldots,\left\langle A_{r}, s\right\rangle\right\}>0$ and

$$
Q^{\prime}=\max \left\{\prod_{\left\{q: s_{q} A_{i q} \neq 0\right\}} Q_{A_{i q}, s_{q} A_{i q}, N}: i=1, \ldots, r\right\}
$$

where $Q_{A_{i q}, s_{q} A_{i q}, N}$ is the constant from Lemma 4.1 for $a=s_{q} A_{i q}, \ell=A_{i q}$ and $h^{\prime}=N$;

(3) a real number $R>1+\frac{T^{c(1+|\mu|)}}{(1-\rho)^{d}}$.

We will prove the following inequalities by induction:

$$
\left|H_{i C D}^{(j)}\right| \leqslant K T^{c|C|} R^{|D|} D !^{t} \prod_{q=1}^{c}\left(|C|+\mu_{q}|D|\right) !^{s_{q}}
$$

for $i=0, \ldots, r, j \in \mathbb{N}$ and $(C, D) \in \mathbb{N}^{c} \times \mathbb{N}^{d}$. Observe that this implies the theorem $\left({ }^{3}\right)$. In fact, for $t^{\prime}=\left(t_{1}+\langle\mu, s\rangle, \ldots, t_{c}+\langle\mu, s\rangle\right)$ and $s^{\prime}=(|s|, \ldots,|s|) \in[0, \infty)^{d}$, the series $\left|\widehat{\mathcal{B}}_{\left(t^{\prime}, s^{\prime}\right)}\right| h_{i}^{(j)}$ defines a real analytic function on $(-\epsilon, \epsilon) \times \stackrel{c+d}{\cdots} \times(-\epsilon, \epsilon)$, whenever

$\left({ }^{3}\right)$ Because the number $\left(|C|+\mu_{1}|D|\right) !^{s_{1}} \cdots\left(|C|+\mu_{c}|D|\right) !^{s_{c}}$ is smaller than or equal to

$$
d^{d\langle s, \mu\rangle} C !^{s^{\prime}} D ! \frac{\langle s, \mu\rangle}{} c^{|s||C|} N^{\langle s, \mu\rangle|D|} .
$$


$\epsilon<\min \left\{1 / T^{c}, 1 / R\right\}$. Then the real analytic functions of one variable

$$
\xi_{i j}(x)=\left|\widehat{\mathcal{B}}_{\left(t^{\prime}, s^{\prime}\right)}\right| h_{i}^{(j)}(x, \ldots, x) \text { for }|x|<\epsilon,
$$

can be factorized as $\xi_{i j}(x)=x^{j} \beta_{i j}(x)$, by the order conditions $\left(\nu_{i j}\right)$. The functions $\beta_{i j}$ are positive and strictly increasing in $(0, \epsilon)$. Moreover, the inequalities $(*)$ imply that there exists a constant $\left({ }^{4}\right) E>0$ such that $\beta_{i j}(x) \leqslant E x^{-j}$ in $(0, \epsilon)$. Hence for $i=0, \ldots, r$ and $j \in \mathbb{N}$ we get

$$
\left\|h_{i}^{(j)}\right\|_{\left(t^{\prime}, s^{\prime}\right), x}=\xi_{i j}(x) \leqslant x^{j} \beta_{i j}(\epsilon) \leqslant E\left(\frac{x}{\epsilon}\right)^{j}, \quad \text { when } x \in(0, \epsilon) .
$$

Consequently $h_{i}=\sum_{j=0}^{\infty} h_{i}^{(j)} \in G^{\left(t^{\prime}, s^{\prime}\right)}$, as we have quoted in the theorem.

In order to prove the inequalities (*), we distinguish two cases. If $j=0$ and $|D| \neq 0$, we have the bounds

$$
\left|H_{i C D}^{(0)}\right| \leqslant \delta D !^{t} \prod_{q=1}^{c}\left(C_{q}+A_{i q}\right) !^{s_{q}} \leqslant K T^{c|C|} R^{|D|} D !^{t} \prod_{q=1}^{c}\left(|C|+\mu_{q}|D|\right) !^{s_{q}} .
$$

Moreover, it is easy to see that

$$
\left|H_{0 C 0}^{(0)}\right| \leqslant K T^{c|C|} \prod_{q=1}^{c}|C| !^{s_{q}} \quad \text { and } \quad\left|H_{i 00}^{(0)}\right| \leqslant K .
$$

In order to achieve the desired inequality for $\left|H_{i C 0}^{(0)}\right|$ we observe that

$$
\begin{aligned}
\left|H_{i C 0}^{(0)}\right| & \leqslant \delta\left(\prod_{\substack{1 \leqslant q \leqslant c \\
s_{q} A_{i q} \neq 0}} T^{C_{q}}\right)\left(\prod_{\substack{1 \leqslant q \leqslant c \\
s_{q} A_{i q} \neq 0}} \frac{\left(C_{q}+A_{i q}\right) !^{s_{q}}}{T^{C_{q}}}\right)\left(\prod_{\substack{1 \leqslant q \leqslant c \\
A_{i q}=0}} C_{q} !^{s_{q}}\right) \\
& \leqslant \delta T^{c|C|}\left(\prod_{\substack{1 \leqslant q \leqslant c \\
s_{q} A_{i q} \neq 0}} \varphi_{i q}\left(m_{i q}\right)\right) C !^{s} \leqslant K|C| !^{|s|} T^{c|C|} .
\end{aligned}
$$

If $j>0$, set

$$
\begin{gathered}
S_{1}=\sum_{k=1}^{r} \sum_{\substack{0 \leqslant U \leqslant C+A_{k} \\
0 \leqslant V \leqslant D, V \neq 0}}\left|F_{k U V}\right| \cdot\left|H_{k, C+A_{k}-U, D-V}^{(j-1)}\right|, \\
S_{2}=\sum_{k=1}^{r} \sum_{\substack{0 \leqslant U \leqslant C+A_{k} \\
|U|>\left|A_{k}\right|}}\left|F_{k U 0}\right| \cdot\left|H_{k, C+A_{k}-U, D}^{(j-1)}\right| .
\end{gathered}
$$

Hence, if we have bounds for $S_{1}$ and $S_{2}$, so we have for $\left|H_{i C D}^{(j)}\right|$. By the induction hypothesis

$$
S_{1} \leqslant \delta K D !^{t} T^{c|C|} R^{|D|} \prod_{q=1}^{c}\left(|C|+\mu_{q}|D|\right) !^{s_{q}} \sum_{k=1}^{r} T^{c\left|A_{k}\right|} \sum_{\substack{0 \leqslant U \leqslant C+A_{k} \\ 0 \leqslant V \leqslant D, V \neq 0}}\left(\frac{\rho}{T}\right)^{|U|} \frac{\rho^{|V|}}{R} .
$$

$\left({ }^{4}\right)$ Observe that it is independent of $i$ and $j$. 
Therefore, as $\rho<1$ and $T>\rho$, we get the inequality

$$
S_{1} \leqslant K T^{c|C|} R^{|D|} \frac{\delta r}{(T-\rho)^{c}} \cdot \frac{T^{c(1+|\mu|)}}{R(1-\rho)^{d}} D !^{t} \prod_{q=1}^{c}\left(|C|+\mu_{q}|D|\right) !^{s_{q}}
$$

Consequently, $S_{1} \leqslant K T^{c|C|} R^{|D|} D !^{t} \prod_{q=1}^{c}\left(|C|+\mu_{q}|D|\right) !^{s_{q}} / 2$. The expression $S_{2}$ is bounded by

$$
\delta K D !^{t} R^{|D|} T^{c|C|} \sum_{k=1}^{r} \sum_{\substack{0 \leqslant W \leqslant C+A_{k} \\|W|<|C|}} \rho^{\left|C+A_{k}-W\right|} \prod_{q=1}^{c} \frac{\left(C_{q}+A_{k q}-W_{q}\right) !^{s_{q}}\left(|W|+\mu_{q}|D|\right) !^{s_{q}}}{T^{|C-W|}} .
$$

When $s_{q} A_{k q}>0$, Lemma 4.1 implies the inequality

$$
\frac{\left(C_{q}+A_{k q}-W_{q}\right) !^{s_{q}}\left(|W|+\mu_{q}|D|\right) !^{s_{q}}}{T^{|C-W|}} \leqslant \frac{Q_{A_{k q}, A_{k q} s_{q}, N}}{T^{s_{q} A_{k q}}}|C-W| !^{s_{q}}\left(|W|+\mu_{q}|D|\right) !^{s_{q}} .
$$

Hence, $S_{2} \leqslant K T^{c|C|} R^{|D|} D !^{t} \prod_{q=1}^{c}\left(|C|+\mu_{q}|D|\right) !^{s_{q}} / 2$, and we get our statement $(*)$.

Remark 4.3. There are some special cases where we can divide without changing the Gevrey ring. For instance:

(1) Case $s=0 \in \mathbb{K}^{c}$. Observe that this improves the result obtained by applying the henselian Weierstrass division theorem to this case (see $[\mathrm{H}]$, page 72 ), since the ring $B=$ $\mathbb{K}[[W]]_{t}$ is henselian and the henselianization of the local ring $B[Z]_{(W, Z)}$ is $\mathbb{K}[[W, Z]]_{(t, 0)}$.

(2) Case when $g$ and the $f_{j}$ are in $\left(\mathbb{K}[[W]]_{t,+}\right)[[Z]]_{0,+}$.

The division and preparation theorems for the Gevrey rings can be obtained as corol$\operatorname{laries}\left({ }^{5}\right)$.

Theorem 4.4 (the division theorem for Gevrey's rings). Let $s=\left(s_{1}, \ldots, s_{n}\right) \in[0, \infty)^{n}$ and $d \in \mathbb{N}, d \neq 0$. Let a formal power series

$$
f=\sum_{j=0}^{\infty} F_{j}\left(X_{1}, \ldots, X_{n-1}\right) X_{n}^{j} \in \mathbb{K}\left[\left[X_{1}, \ldots, X_{n}\right]\right]_{s}
$$

satisfy $F_{j}(0, \ldots, 0)=0$ for $0 \leqslant j \leqslant d-1$, and $F_{d}(0, \ldots, 0) \neq 0$. Let $\widehat{s^{\prime}}=\left(s_{1}+d s_{n}, \ldots\right.$ $\left.\ldots, s_{n-1}+d s_{n}\right)$ and $s^{\prime}=\left(\widehat{s}^{\prime}, s_{n}\right)$. Then for each $g \in \mathbb{K}\left[\left[X_{1}, \ldots, X_{n}\right]\right]_{s}$ there exist $q \in$ $\mathbb{K}\left[\left[X_{1}, \ldots, X_{n}\right]\right]_{s^{\prime}}$ and $r \in \mathbb{K}\left[\left[X_{1}, \ldots, X_{n-1}\right]\right]_{\widehat{s^{\prime}}}\left[X_{n}\right]$ such that

$$
g=q f+r \quad \text { and } \quad \operatorname{deg}_{X_{n}} r \leqslant d-1 .
$$

Recall that a formal power series $f\left(X_{1}, \ldots, X_{n}\right) \in \mathbb{K}\left[\left[X_{1}, \ldots, X_{n}\right]\right]$ is $X_{n}$-regular of order $d$ if the order of $f\left(0, \ldots, 0, X_{n}\right) \in \mathbb{K}\left[\left[X_{n}\right]\right]$ is $d$ for the valuation given by the maximal ideal of $\mathbb{K}\left[\left[X_{n}\right]\right]$. Moreover, we say that $f$ is $X_{n}$-regular, if it is $X_{n}$-regular of order $\operatorname{ord}_{0} f$.

$\left({ }^{5}\right)$ See [Z2] for a simpler proof. 
Theorem 4.5 (the preparation theorem for Gevrey's rings). Let $f \in \mathbb{K}\left[\left[X_{1}, \ldots, X_{n}\right]\right]_{s}$ for some $s=\left(s_{1}, \ldots, s_{n}\right) \in[0, \infty)^{n}$, and $d=\operatorname{ord}_{0}(f)$. If $f$ is $X_{n}$-regular, then there exist a unit $u \in \mathbb{K}\left[\left[X_{1}, \ldots, X_{n}\right]\right]_{s^{\prime}}$ and $a_{1}, \ldots, a_{d-1}$ in $\mathbb{K}\left[\left[X_{1}, \ldots, X_{n-1}\right]\right]_{\widehat{s}^{\prime}}$ such that

$$
f=u \cdot\left(X_{n}^{d}+\sum_{j=0}^{d} a_{j} X_{n}^{j}\right)
$$

where $\widehat{s^{\prime}}=\left(s_{1}+d s_{n}, \ldots, s_{n-1}+d s_{n}\right)$ and $s^{\prime}=\left(\widehat{s^{\prime}}, s_{n}\right)$.

Now, fix independent variables $X_{1}, \ldots, X_{n}$ over $\mathbb{K}$, and consider the ring

$$
\mathbb{G}=\bigcup_{s \geqslant 0} G^{(s)} .
$$

We call it the Gevrey ring (of dimension $n$ ). In fact, $\mathbb{G}$ is a local regular ring of dimension $n$ and it is also a henselian unique factorization domain. The normalization theorem can be proved for $\mathbb{G}$ analogously to the case of the convergent power series rings $([\mathrm{N}])$. Moreover it is an excellent ring (see $[\mathrm{Mt}]$, page 291). So, it has the approximation property, by $[\mathrm{R}]$, that is:

TheOREM 4.6. Given a finite family of polynomials $P_{i}\left(Y_{1}, \ldots, Y_{m}\right)$ in $\mathbb{G}\left[Y_{1}, \ldots, Y_{m}\right]$, for $i=1, \ldots, r$, and a formal solution $\left(\widehat{y}_{1}, \ldots, \widehat{y}_{m}\right)$ in $\mathbb{K}\left[\left[X_{1}, \ldots, X_{n}\right]\right]^{m}$ of the system

$$
P_{i}\left(Y_{1}, \ldots, Y_{m}\right)=0, \quad i=1, \ldots, r,
$$

there exists a solution $\left(y_{1}, \ldots, y_{m}\right)$ of $(*)$ in $\mathbb{G}^{m}$.

Similarly to the convergent power series rings, we have in $\mathbb{G}$ a theorem of implicit series and a theorem of inverse series. More precisely, we have the following results:

Theorem 4.7 (the implicit series theorem). Let $n, m$ be two positive integers, and $X_{1}, \ldots, X_{n}, Z_{1}, \ldots, Z_{m}$ independent variables over $\mathbb{K}$. Given $s=\left(s_{1}, \ldots, s_{n+m}\right)$ in $[0, \infty)^{n+m}$, we put $r=\max \left\{s_{i}: 1 \leqslant i \leqslant n+m\right\}$. Let

$$
f_{j}\left(X_{1}, \ldots, X_{n}, Z_{1}, \ldots, Z_{m}\right) \in \mathbb{K}\left[\left[X_{1}, \ldots, X_{n}, Z_{1}, \ldots, Z_{m}\right]\right]_{s} \quad \text { for } j=1, \ldots, n,
$$

with $\operatorname{det}\left(\frac{\partial f_{i}}{\partial X_{j}}(0)\right)_{i, j} \neq 0$ and $f_{j}(0)=0$. Then there exist $\xi_{1}, \ldots, \xi_{n}$ in $\mathbb{K}\left[\left[Z_{1}, \ldots, Z_{m}\right]\right]_{2^{n} \underline{r}}$ such that

$$
f_{j}\left(\xi_{1}, \ldots, \xi_{n}, Z_{1}, \ldots, Z_{m}\right)=0 \quad \text { for } j=1, \ldots, n \text {. }
$$

Proof. For $n=1$ the statement is an easy consequence of the division theorem (Thm. 4.4). We can suppose that $\frac{\partial f_{n}}{\partial X_{n}}(0) \neq 0$, and then we have the equalities

$$
\begin{aligned}
f_{n} & =u \cdot\left(X_{n}-\xi\left(X_{1}, \ldots, X_{n-1}, \underline{Z}\right)\right), \\
f_{j} & =q_{j} f_{n}+r_{j}\left(X_{1}, \ldots, X_{n-1}, \underline{Z}\right) \text { for } j=1, \ldots, n-1,
\end{aligned}
$$


with $\xi, r_{j}$ in $\mathbb{K}\left[\left[X_{1}, \ldots, X_{n-1}\right]\right]_{2 \underline{r}}$. Moreover, we get

$$
\begin{aligned}
& \frac{\partial f_{n}}{\partial X_{n}}(0) \neq 0, \\
& \frac{\partial f_{j}}{\partial X_{n}}(0)=q_{j}(0) \frac{\partial f_{n}}{\partial X_{n}}(0) \quad \text { for } j=1, \ldots, n-1, \\
& \frac{\partial f_{j}}{\partial X_{k}}(0)=q_{j}(0) \frac{\partial f_{n}}{\partial X_{k}}(0)+\frac{\partial r_{j}}{\partial X_{k}}(0) \quad \text { for } j=1, \ldots, n-1 .
\end{aligned}
$$

The matrix $\left(\frac{\partial r_{j}}{\partial X_{k}}(0)\right)_{j, k}$ is invertible. Hence, by induction, there exist $\xi_{1}, \ldots, \xi_{n-1}$ in $\mathbb{K}[[\underline{Z}]]_{2^{n-1} \underline{\underline{r}}}$ such that $r_{j}\left(\xi_{1}, \ldots, \xi_{n-1}, \underline{Z}\right)=0$. Now put $\xi_{n}=\xi\left(\xi_{1}, \ldots, \xi_{n-1}, \underline{Z}\right)$. This is an element of $\mathbb{K}[[\underline{Z}]]_{2^{n-1} \underline{r}}$, by the evaluation property. So, $\xi_{1}, \ldots, \xi_{n}$ are the required series.

Lemma 4.8. Let $X_{1}, \ldots, X_{n}, Z_{1}, \ldots, Z_{n}$ be independent variables over $\mathbb{K}$, and $\mathbb{G}$ the Gevrey ring of dimension $n$ in the variables $X_{j}$. Let $f_{1}, \ldots, f_{n} \in \mathbb{K}\left[\left[X_{1}, \ldots, X_{n}\right]\right]_{\left(s_{1}, \ldots, s_{n}\right)}$ be such that the determinant $\operatorname{det}\left(\frac{\partial f_{i}}{\partial X_{j}}(0)\right) \neq 0$. Put $r=\max \left\{s_{1}, \ldots, s_{n}\right\}$. Then there exist $\xi_{1}, \ldots, \xi_{n}$ in $\mathbb{K}[[\underline{Z}]]_{2^{n} \underline{\underline{r}}}$ such that $f_{j}\left(\xi_{1}, \ldots, \xi_{n}\right)=Z_{j}$ for $j=1, \ldots, n$.

Proof. Take $g_{j}=f_{j}-Z_{j}$ and apply the implicit series theorem.

We have the following corollary to the previous lemma:

Theorem 4.9 (the inverse series theorem). Let $f_{1}, \ldots, f_{n} \in \mathbb{K}\left[\left[X_{1}, \ldots, X_{n}\right]\right]_{\left(s_{1}, \ldots, s_{n}\right)}$ be such that $\operatorname{det}\left(\frac{\partial f_{i}}{\partial X_{j}}(0)\right) \neq 0$. Put $r=\max \left\{s_{1}, \ldots, s_{n}\right\}$. Then there exist $\xi_{1}, \ldots, \xi_{n}$ in $\mathbb{K}[[\underline{Z}]]_{2^{n} \underline{r}}$ such that $f_{j}\left(\xi_{1}, \ldots, \xi_{n}\right)=X_{j}$ for $j=1, \ldots, n$.

\section{References}

[ADM] G. R. Allan, H. G. Dale, J. P. McClure, Pseudo-Banach algebras, Studia Math. 40 (1971), 55-69.

[AHV] J. M. Aroca, H. Hironaka, J. L. Vicente, The Theory of the Maximal Contact, Memorias de Matemática del Instituto "Jorge Juan" 29, Madrid, 1975.

[BR] R. Benedetti, J. J. Risler, Real Algebraic and Semi-algebraic Sets, Actualités Math., Hermann, Paris, 1990.

[B] J. W. Brewer, Power Series over Commutative Rings, Lecture Notes in Pure and Appl. Math. 64, Marcel Dekker, New York, 1981.

[Ca] J. Cano, On the series defined by differential equations with an extension of the Puiseux polygon construction to this series, Analysis 13 (1993), 103-119.

[CC1] J. Chaumat, A. M. Chollet, Sur le théorème de division de Weierstrass, Studia Math. 116 (1995), 59-84.

[CC2] J. Chaumat, A. M. Chollet, Théorème de preparation dans les classes ultradifferentiables, C. R. Acad. Sci. Paris Sér. I Math. 320 (1995), 1305-1310.

[Co] P. M. Cohn, Puiseux's theorem revisited, J. Pure Appl. Algebra 31 (1984), 1-4.

[G] M. Gevrey, Sur la nature analytique des solutions des équations aux dérivées partielles, Ann. Sci. École Norm. Sup. (3) 25 (1918), 129-190.

$[\mathrm{H}]$ H. Hironaka, Idealistic exponents of singularity, in: Algebraic Geometry, John Hopkins Univ. Press, Baltimore, 1977, 52-125. 
[Mi] E. Maillet, Sur les séries divergentes et les équations differentielles, Ann. Sci. École Norm. Sup. 3 (1903), 487-518.

[Ml] B. Malgrange, Sur le théorème de Maillet, Asymptot. Anal. 2 (1989), 1-4.

[M] H. Matsumura, Commutative Algebra, Math. Lecture Note Ser. 56, Benjamin/ Cumming Publishing Co., Reading, 1980.

[N] M. Nagata, Local Rings, Robert E. Krieger Publishing Co., Huntington, 1975.

[O] S. Ouchi, Formal solutions with Gevrey type estimates of nonlinear partial differential equations, J. Math. Sci. Univ. Tokyo 1 (1994), 205-237.

[R] C. Rotthaus, On the approximation theory of excellent rings, Invent. Math. 88 (1987), 39-63.

[T] J. Cl. Tougeron, Sur les ensembles semi-analytiques avec conditions Gevrey au bord, Ann. Sci. École Norm. Sup. (4) 27 (1994), 173-208.

[Z1] M. A. Zurro, Le théorème de division pour les séries Gevrey à plusieurs variables, Preprint, University of Valladolid, Spain, 1992.

[Z2] M. A. Zurro, The Abhyankar Jung theorem revisited, J. Pure Appl. Algebra 90 (1993), $257-282$.

[Z3] M. A. Zurro, Series y funciones Gevrey en varias variables, Ph.D. Thesis, University of Valladolid, Spain, 1994.

[Z4] M. A. Zurro, Summability "au plus petit terme", Studia Math. 113 (1995), 197-198. 